\title{
Assimilation, Integration, Kohäsion, Partizipation - oder: Was wird hier eigentlich verhandelt?
}

\author{
Walter Leimgruber
}

In der politischen und medialen Diskussion werden Migration, die Herausforderungen der wachsenden Mobilität und die Chancen und Risiken von Integration pausenlos verhandelt. Doch müsste von etwas viel Grundsätzlicherem die Rede sein, nämlich von der Ausgestaltung demokratischer und staatlicher Macht, von Kohäsion und Partizipation angesichts vielfacher Herausforderungen zunehmend mobiler und global verfasster Gesellschaften. Denn diese Herausforderungen stellen die bisherigen Prinzipien demokratischer Teilhabe auf nationalstaatlicher Ebene in Frage und verlangen nach neuen Lösungen. ${ }^{1}$

\section{VON ASSIMILATION BIS TRANS}

Lange Zeit war die Ausgangslage klar: Die Migrantinnen und Migranten sind Bittstellende, die sich an die Anforderungen der Gesellschaften, in die sie einwandern anzupassen haben. In Einwanderungsnationen wie den USA wurde das Bild des „melting pot“ kreiert, des Schmelztiegels, der die unterschiedlichen Herkunftskulturen zu einer neuen Kultur vereinte. Diese Vorstellung wurde allerdings im Laufe der Zeit in Frage gestellt, weil die Akzeptanz verschiedener kultureller Lebensweisen in der Realität sehr unterschiedlich war und nicht alle Gruppen in einem

1 Die Fussnoten enthalten aufgrund des beschränkten Platzes wenige weiterführende Literaturhinweise. Teile der Argumentation finden sich auch in früheren Aufsätzen von mir. 
homogenen amerikanischen Lebensstil aufgingen, was mit den Bildern der ,salad bowl“" oder des ,cultural mosaic" beschrieben wurde. ${ }^{2}$

In europäischen Staaten, die sich nicht als Einwanderungsländer definierten, dominierte das Bild der Assimilation, verstanden als einseitige Anpassung. Kultur wird dabei - bildlich gesprochen - als eine Art geistiges Gepäckstück, als kompakter Mentalrucksack verstanden, den man in ein neues gesellschaftliches Umfeld mitbringt, dort in den Schrank stellt und durch einen neuen ersetzt. Das Problem dieses Konzepts liegt in der Frage, wie man eine als homogen verstandene Kultur einfach ablösen und ersetzen kann. Die Vertreterinnen und Vertreter dieser Konzepte in der Politik betonen, wenn es um ihre eigene Kultur geht, dass diese eine traditionsreiche und tief verwurzelte Sache sei, die es zu respektieren gelte, verlangen aber von anderen, diese Verwurzelung sei aufzugeben und durch eine vollständig neue Kulturpackung zu ersetzen. Aus diesem Grund ist das Assimilationskonzept im Laufe der Forschung als gescheitert betrachtet worden, um aber in den letzten Jahren mit einer Reihe von Anpassungen erneut diskutiert zu werden. $^{3}$

Der Begriff Integration, der den Assimilationsbegriff ablöste und für lange Zeit die Diskussion beherrschte, wird von manchen Autoren und Autorinnen ähnlich oder gleich wie der Begriff der Assimilation verwendet. Wissenschaftlich unterscheiden sich Integrationsansätze aber vom Assimilationskonzept, weil sie nicht von einer „kugelförmigen“, einheitlichen und integralen Kultur ausgehen, sondern mit dem Modell einer differenzierten und segmentierten modernen Gesellschaft arbeiten. ${ }^{4}$ In dieser sind die Individuen in verschiedenen Bereichen aktiv und müssen sich primär auf jenen gesellschaftlichen Ebenen integrieren, die für sie wichtig sind: Für die Arbeitsmigrantin ist dies ein anderer Ort als für den politischen Flüchtling, für den Unternehmer ein anderer als für die Wissenschaftlerin. Nicht die Gesellschaft als Gesamtheit mit ihrer wie auch immer verstandenen Kultur, sondern die Teilhabe an einzelnen Bereichen bildet den Ausgangspunkt der Integration. Diese ist daher ein vielschichtiger Prozess, der sich auf mehreren Ebe-

2 D'Innocenzo, Michael/Sirefman, Josef P. (eds.): Immigration and Ethnicity. American Society - >Melting Pot $\prec$ or `Salad Bowk, New York: Greenwood 1992.

3 Alba, Richard/Nee, Victor: Remaking the American Mainstream. Assimilation and Contemporary Immigration, Cambridge, Mass.: HUP 2003; Koopmans, Ruud: Assimilation oder Multikulturalismus? Bedingungen gelungener Integration, Münster: Lit 2017.

4 Grundlegend dazu immer noch die Arbeiten von Hartmut Esser, z.B.: Integration und ethnische Schichtung (= MZES Arbeitspapiere Nr. 40). Mannheim 2001. 
nen und in unterschiedlichen Tempi und Gewichtungen vollzieht und zu unterschiedlichsten Verlaufsformen führt.

Als weiteres Element enthalten Integrationskonzepte häufig den Hinweis, dass Integration Aufgabe der gesamten Gesellschaft und nicht nur der Zugewanderten ist, und dass beide Seiten zu lernen und sich in ihren strukturellen Voraussetzungen anzupassen haben. In der Schweiz wurde lange Zeit von „fördern und fordern“ gesprochen, um Anspruch wie Erwartung zu betonen, auch das in der Regel auf alle involvierten Parteien bezogen. ${ }^{5}$

Von vielen Migrantinnen und Migranten und v. a. auch von Angehörigen der zweiten Generation wird der Begriff „Integration“ heute abgelehnt, auch im Fach ist die Skepsis gross. ${ }^{6}$ Immer häufiger findet sich die Bezeichnung in der Tat in Gesetzen, welche die Integrationsanforderungen für Migrierende definieren und die primär als Druckmittel eingesetzt werden. Deshalb wirkt „Integration“ politisch oft nur als einseitiger Forderungskatalog. Sie benötigt aber zwei Seiten, Migrierende können sich nicht integrieren ohne Gegenseite, die ihrerseits ihre Strukturen und Prozesse anpasst. Und sie benötigt zwei Ebenen, die der Verpflichtung und die der Förderung. Ohne fördernde Massnahmen zwischen Sprachunterricht und Diskriminierungsschutz funktioniert Integration nicht, das Konzept „fördern und fordern“ galt ursprünglich daher für die lokale Gesellschaft und deren Institutionen genauso wie für die Migrierenden, was heute häufig ausgeblendet wird. ${ }^{7}$

Das Konzept der Multikulturalität galt lange als Gegenentwurf zum Assimilationsansatz. Es thematisiert die Verschiedenheit von Kulturen innerhalb eines Staats bei gleichzeitiger Gewährleistung individueller Rechte durch den Staat. In seiner klassischen Form geht es von einer Existenz deutlich unterscheidbarer, in sich homogener, ethnisch definierter Kulturen in einer Gesellschaft gleicher

5 Ehret, Rebekka: Leitbild und Handlungskonzept des Regierungsrates zur Integrationspolitik des Kantons Basel-Stadt, Basel 1999; Sancar-Flückiger, Annemarie: »Integrationsleitbilder und Integrationspolitik: zur kontroversen Leitbild-Debatte in Zürich, Bern und Basel«, in: Dietrich, Helmut et al. (Hg.): Flüchtlinge, Migration und Integration, Zürich: Widerspruch 1999, S. 137-145.

6 Hess, Sabine/Binder, Jana/Moser, Johannes (Hg.): No integration?! Kulturwissenschaftliche Beiträge zur Integrationsdebatte in Europa, Bielefeld: Transcript 2009; Schinkel, Willem: Imagined Societies. A Critique of Immigrant Integration in Western Societies, Cambridge: CUP 2017.

7 Eidgenössische Migrationskommission (EKM): Integration - kein Messinstrument, sondern die Aufgabe aller. Empfehlungen, 18.12.2017. https://www.ekm.admin.ch/ dam/data/ekm/dokumentation/empfehlungen/empf_integration_aufgabe_d.pdf (16.3.2019). 
Bürgerinnen und Bürger aus. Jede Kultur bekommt Gestaltungsraum zuerkannt, so dass auf der Basis gegenseitiger Toleranz ein Miteinander, häufig auch eher ein Nebeneinander geschaffen wird, das von der Unterscheidung „öffentlich“ und „privat“" lebt. Während im öffentlichen Bereich allgemeingültige Regeln gelten, sind im privaten Bereich vielfältige Lebensformen akzeptiert. ${ }^{8}$

Die Trennung zwischen einem öffentlichen Bereich, in dem gegenseitige Toleranz vorausgesetzt wird, und gleichzeitiger privater kultureller Freiheit funktioniert allerdings nicht. Denn diese Trennung ist ein spezifisch westliches Konstrukt, das in anderen Gesellschaften meist nicht existiert. Zudem ist in vielen Fällen nicht klar zuordenbar, ob bestimmte Verhaltensweisen in den öffentlichen oder privaten Bereich gehören, etwa bei der einfach erscheinenden Frage, welche Kleidung man trägt. Kritisiert wird weiter, dass die Grundlage des Konzepts ein statischer Kulturbegriff ist, der die einzelnen Personen zu Gefangenen ihrer ethnischen Herkunftskulturen macht und sich damit kaum vom Kulturkonzept der Assimilationstheorie unterscheidet. Machtwirksame Aspekte der sozialen und der ökonomischen Stellung oder des Geschlechts bleiben weitgehend ausgeblendet. ${ }^{9}$

Die nächste Phase der Forschung konzentrierte sich vermehrt auf den Austausch, auf transnationale und transkulturelle Beziehungsebenen, die durch die Migration entstehen. Beziehungsnetze, Produktion, Konsum und Politik lassen prozessuale „Landschaften“ entstehen, die global miteinander verbunden sind, ohne dass noch territoriale Zusammenhänge bestehen. ${ }^{10}$ Auch auf der Ebene der Identität müssen sich die Individuen mit vielen Aspekten der Zugehörigkeit - sozial, kulturell, geschlechtlich, bildungsbezogen, berufsmässig etc. - auseinandersetzen, die nicht einfach zugewiesen oder von Generation zu Generation weiter gegeben werden, sondern gewählt werden können oder müssen. Sie werden zu „Touristen“" und „Vagabunden“ (Zygmunt Bauman). ${ }^{11}$

8 Baumann, Gerd et al. (eds.): Multiculturalism. Critical concepts in Sociology. 4 vols. London: Routledge 2011.

9 Neubert, Stefan et al. (Hg.): Multikulturalität in der Diskussion. Neuere Beiträge zu einem umstrittenen Konzept, Wiesbaden: Springer 2013.

10 Bauböck, Rainer/Feist, Thomas (eds.): Diaspora and Transnationalism. Concepts, Theories and Methods, Amsterdam: AUP 2010; Appadurai, Arjun: »Global Ethnoscapes: Notes and Queries for a Transnational Anthropology«, in: Fox, Richard G. (Ed.): Recapturing Anthropology. Working in the Present, Santa Fe: School of American Research Press 1991, pp. 191-210.

11 Hall, Stuart et al. (eds.): Questions of Cultural Identity, London: Sage 2011; darin v. a. Bauman, Zygmunt: »From Pilgrim to Tourist or a Short History of Identity«, pp. 1836. 
Transnationale Theorien verweisen darauf, dass Migration ein Netzwerk erzeugt, das sich zwischen Staaten und über staatliche Strukturen und Gesellschaften hinweg aufspannt und zugleich deren Strukturen verändert. ${ }^{12}$

\section{ZUGEHÖRIGKEITEN, WERTE UND WANDEL}

Anders als von vielen Forschenden angenommen, spielt der Staat aber weiterhin eine wichtige Rolle und wird nicht verschwinden, denn auch für viele Migrierenden bleiben die Staatsbürgerrechte eine zentrale Grösse ihrer sozialen Positionierung. Zwar beobachten wir eine Flexibilisierung und Öffnung, erkennbar etwa in der zunehmenden Akzeptanz der mehrfachen Staatsbürgerschaft oder im gemeinsamen Raum der EU-Bürgerschaft. Über den Nationalstaat legen sich also neue Ebenen der Zugehörigkeit. Doch in welchen transnationalen „Räumen“ Migrierende auch immer leben, ein quasi freischwebender, vom Staat vollständig losgelöster Zustand ist nicht zu erkennen.

Und es ist gerade diese Zugehörigkeit, die eine andere Perspektive als die Dualisierung zwischen Migrierenden auf der einen und Herkunfts- wie Ankunftsgesellschaft auf der anderen Seite nahelegt, die den meisten Migrationstheorien inhärent ist, nämlich einen gesamtgesellschaftlichen Blick, der alle gleichermassen betrifft: Einen Kohäsionsprozess, der Zugehörigkeit erzeugt. Die entsprechenden Bemühungen sind dabei nicht ausschliesslich auf Migrierende ausgerichtet, sondern auf alle, die in vielfältigen Konstellationen und Zugehörigkeiten und auch in vielfältigen Mobilitäten leben. Kohäsion betrifft in heutigen ebenso diversen wie mobilen Gesellschaften alle. ${ }^{13}$ Alle, die in einem Staat zusammenleben, aber auch alle, die in und zwischen mehreren Staaten leben, so dass sich das Modell singulärer und ausschliesslicher Zugehörigkeit zunehmend auflöst.

Was wir aktuell in den meisten westlichen Gesellschaften erleben, ist allerdings eine sehr spezifische Art dieses Kohäsionsprozesses, eine Art regressive und rückwärtsgewandte Kohäsion, die angesichts verschiedenster Herausforderungen versucht, das kohäsive Element wieder stärker zu betonen und dabei v. a. auf zwei Elemente setzt: Erstens auf Rückwärtsgewandtheit und Orientierung an einem

12 Levitt, Peggy/Jaworsky, Nadya: »Transnational Migration Studies: Past Developments and Future Trends«, in: Annual Review of Sociology 33 (2007), pp. 129-156.

13 Vgl. zur Diskussion um Kohäsion: Schiefer, David/Noll, Jolanda: »The Essentials of Social Cohesion. A Literature Review«, in: Social Indicators Research: An International and Interdisciplinary Journal for Quality-of-Life Measurement 132/2 (2017), pp. 579-603. 
angeblichen historischen Idealzustand, den es in Wirklichkeit so nie gegeben hat und der nur dargestellt werden kann unter Ausblendung historischer Ausschlussprozesse wie auch früherer Mobilitäten und Wanderungsbewegungen. ${ }^{14}$ Und zweitens auf den Ausschluss von bestimmten Gruppen, nicht nur, aber auch von Migrierenden bzw. von spezifischen migrantischen Gruppen. Aus Furcht vor Krisen, Bedeutungsverlust oder Terrorbedrohung werden ordnungs- und sicherheitspolitische Forderungen nach Renationalisierung und Grenzschliessung aufgestellt. Diese Suche nach Zusammenhalt führt daher zur Suche nach Stützen, an denen man sich festhalten kann, etwa zur Suche nach Heimat, die so sehr im Trend liegt, dass sogar „Heimatministerien“ benötigt werden.

In einer klaren Abgrenzung vom „Fremden“ soll definiert werden, welche Werte Allgemeingültigkeit besitzen. ${ }^{15}$ Dabei wird häufig übersehen, dass Wertedebatten nicht einfach mit „eigen“ und „fremd“, mit Migration und Globalisierung zusammenhängen, sondern grundlegendes Element jeder Gesellschaft sind. Vieles ist heute selbstverständlich, was eben noch heftig umstritten war. Man denke etwa an den schnellen Bedeutungsverlust der Religion, die sich wandelnden Geschlechterrollen oder die Einstellung zu gleichgeschlechtlicher Liebe; alles Bereiche, die wenig mit Migration und Globalisierung zu tun und die sich in den letzten Jahren grundlegend verändert haben. Die Vorstellung des Schaffens eines Konsenses gültiger Werte übersieht den Wandel und betont zu sehr die historische Kontinuität. Und sie übersieht, dass gerade die konstante Auseinandersetzung um Werte wesentliches Element demokratischer gesellschaftlicher Kohäsion ist, so dass Werte nicht einfach verordnet oder stabil gehalten werden können. Allerdings kann diese grundlegende Debatte dann problematisch werden, wenn die Menge der Änderungen und Herausforderungen so gross wird, dass sie primär Angst vor der Zukunft erzeugt. In diesem Fall entsteht eine gesellschaftliche Schliessungsbewegung, die sich wiederum auf angeblich schon immer gültige Werte beruft, wie wir das aktuell erleben. Sichtbar wird dabei das Gefühl von Zerrissenheit der Gesellschaft einerseits, des Individuums andererseits. Dieses Gefühl, das heute weite Teile der Gesellschaft erfasst hat, kennen ausgerechnet Migrantinnen und Migranten bestens. Aus der Auseinandersetzung zwischen Herkunftsgebiet und

14 Leimgruber, Walter: »Constructing a Home. Heimat as Expression of Privilege, Belonging, Exclusion, and Identity«, in: Moser, Johannes et al. (eds.): Ways of Dwelling. Crisis - Craft - Creativity, Journal for European Ethnology and Cultural Analysis, Supplement, vol. 1, 2019, pp. 142-167.

15 Zimmermann, Olaf/Geissler, Theo: Wertedebatte. Von Leitkultur bis kulturelle Integration, Berlin: Deutscher Kulturrat 2018; Sommer, Andreas Urs: Werte. Warum man sie braucht, obwohl es sie nicht gibt, Metzler: Stuttgart 2016. 
neuem Lebensmittelpunkt ergibt sich für sie häufig eine Konstellation des Zerrissenseins zwischen den Wertvorstellungen und kulturellen Erwartungen von ,hier“ und von „dort“. Offensichtlich ist es in den letzten Jahrzehnten zu einer Art Migrantisierung der gesamten Gesellschaft gekommen. Durch verschiedene gesellschaftlichen Entgrenzungsprozesse sind die Erfahrungen der Zerrissenheit allgemein geworden. Dem ,schwachen“ Migrierenden von damals stehen daher nicht mehr der starke Staat und die selbstbewusst auftretende Gesellschaft der Assimilations- und Integrationsära gegenüber, sondern ein zutiefst verunsichertes Gemeinwesen. Wenn ich von regressiver Kohäsion spreche, meine ich die gesellschaftliche Suche nach Lösungen in vielen Bereichen, bei denen gerne zuerst die Migration konkret oder die Globalisierung abstrakt verantwortlich gemacht wird. In Wirklichkeit aber sind Prozesse am Werk, die auf die gesamte Gesellschaft einwirken und die nicht einfach eine Ursache haben: Da ist etwa der Wandel in der Arbeitswelt, das Verschwinden von ganzen Arbeitsbereichen und die Verschiebung der Produktion von Gütern und Dienstleistungen in Länder, in denen Lohnund Produktionskosten tief sind. ${ }^{16}$ Eine weitere Baustelle betrifft die sich grundlegend verändernde demographische Ordnung. Es gilt neue Lösungen zu finden für das Verhältnis von Arbeitsplätzen und Rentensystem, von schwindender Bevölkerung und Arbeitskräftebedarf. ${ }^{17}$ Eine dritte fundamentale Veränderung stellen die Gleichstellung der Geschlechter und neue Partnerschafts- und Familienmodelle dar, die dazu führen, dass die klassische Einpersonen-Ernährer-Familie verschwinden wird und für die Betreuung von Kindern und Alten neue Lösungen gesucht werden müssen. ${ }^{18}$

Solche Veränderungen rufen immer Ängste und Gegenreaktionen hervor. Erfolgreich können sie nur dann sein, wenn sie die Menschen nicht nur verunsichern, sondern auch eine Zukunftsperspektive bieten. Nur dann kann ein Kohäsionsprozess entstehen, der nicht repressiv ist, sondern progressiv, indem er versucht, neue Entwicklungen ebenso wenig auszuschliessen wie spezifische Gruppen, die für

16 Sweet, Stephen/Meiksins, Peter: Changing Contours of Work. Jobs and Opportunities in the New Economy, Los Angeles: Sage 2017.

17 Véron, Jacques/Pennec, Sophie/Legare, Jacques (eds.): Ages, Generations and the Social Contract. The Demographic Challenges Facing the Welfare State, Dordrecht: Springer 2007.

18 Nadai, Eva/Nollert, Michael (Hg.): Geschlechterverhältnisse im Post-Wohlfahrtsstaat, Weinheim: Beltz Juventa 2015; Esping Andersen, Gøsta: »A Social Model for MidCentury Europe«, in: Bonoli, Giuliano/Bertozzi, Fabio (éds.): Les nouveaux défis de l'Etat social, Lausanne: Presses polytechniques et universitaires romandes 2008, pp. $235-252$. 
diese verantwortlich gemacht werden. Ein Prozess also, der Kohäsion inkludierend statt exkludierend herstellt und die Partizipation möglichst aller Mitglieder einer Gesellschaft anstrebt.

\section{WELCHE RECHTE FÜR WELCHE GRUPPEN?}

Bevor wir uns mit den Herausforderungen der Partizipation beschäftigen, gilt es eine grundlegende Problematik moderner Gesellschaften in den Blick zu nehmen: Was deren Mitgliedern als Rechte und Pflichten zugestanden wird, hat einen entscheidenden Wandel erfahren, was die Zuordnung dieser Rechte und Pflichten zu bestimmten Gruppen betrifft: Denn diese Ebenen haben sich im Laufe der Herausbildung moderner National- und Sozialstaaten unterschiedlich entwickelt: Die Aufklärung und die Französische Revolution brachten erstens die Idee der Menschenrechte, der Gewissens-, Meinungs-, Rede-, der Versammlungsfreiheit, die zentral sind für die Herausbildung der westlichen Gesellschaft, auch wenn ihre Durchsetzung lange gedauert hat und immer wieder gefährdet ist. Länger dauerte es zweitens mit den politischen Rechten, dem demokratischen Stimm- und Wahlrecht, das vielerorts erst im 20. Jahrhundert durchgesetzt wurde. Und schliesslich kamen drittens die sozialen Rechte hinzu, die mit dem Ausbau der Sozialstaaten entstanden und die Individuen absichern mit Arbeitslosen-, Kranken- und Rentenkassen.

Die bürgerlichen, die politischen und die sozialen Rechte bilden seit dem Beginn des Sozialstaates die Trias der staatlichen Zugehörigkeit, erklärte Thomas H. Marshall in seinem berühmten Essay von 1950. ${ }^{19}$ Über lange Zeit hinweg wurden sie nationalstaatlich gedacht, geschaffen mit Blick auf die Staatsbürgerinnen und -bürger. Heute aber gelten sie für viele weitere Menschen. Wir sind daher auf den unterschiedlichsten Ebenen mit Rechten ausgestattet. Die politischen Rechte gehören noch immer den Staatsbürgerinnen und -bürgern der einzelnen Staaten. Die Menschenrechte hingegen wurden zu universell geschützten Rechten, sind nicht an einen bestimmten legalen Status des Individuums gebunden. Viele davon werden nicht mehr durch staatliches Recht geregelt, sondern durch Vereinbarungen auf internationaler Ebene.

Die sozialen Rechte wiederum gehören einer anders definierten Gruppe, die in einem Land einen bestimmten Aufenthaltsstatus besitzt, der jedoch nicht von der Staatsbürgerschaft abhängig ist. Es entstand daher ein Zwischenstatus zwischen

19 Marshall, Thomas H.: Citizenship and Social Class and Other Essays, Cambridge: CUP 1950. 
Staats- und Weltbürger, etwa „,denizen“ oder „Wohnbürger“20 genannt. Gleichzeitig werden aber die entscheidenden Weichenstellungen gerade im Bereich der Sozialpolitik von den Staatsangehörigen getroffen, wesentliche Teile der Betroffenen ausgeschlossen. Die Einheit von Staat, Recht und Bürgerin bzw. Bürger ist nicht mehr gegeben. Das heisst, dass die Regeln der Partizipation - wer hat das Recht, sich wann und in welchem Rahmen am politischen Entscheidungsprozess zu beteiligen - neu gedacht werden müssen.

Die grösste Attraktivität der europäischen Staaten liegt nach Ansicht vieler aber nicht in der Möglichkeit politischer Partizipation, sondern in der Zugehörigkeit zu einem sozialstaatlichen Netz. Dieses wird durch die Zuwanderung zunehmend strapaziert, lautet eine populäre Behauptung migrationsskeptischer Gruppen. Migration und Ausbau des Sozialstaats sind in der Tat zwei widerstreitende Entwicklungen der Moderne. ${ }^{21}$ In der Frühen Neuzeit waren die Gemeinden zuständig für die so genannte Armenfürsorge. Arme, die kein Gemeindebürgerrecht besassen, wurden vertrieben; die Niederlassungsfreiheit war unbekannt. Mit der Nationalstaatenbildung wurde erstmals die freie Mobilität innerhalb staatlicher Grenzen möglich. Im Laufe der Zeit entwickelten alle westlichen Staaten ein immer dichteres Netz sozialer Abfederung. Neben Steuern, Militärdienst und demokratischen Rechten entstand damit eine weitere Ebene der Verbindung zwischen dem Staat und seinen Bürgerinnen und Bürgern, die den Prozess der Binnenintegration förderte. Im gleichen Zeitraum wurde die Grenz- und Einwanderungspolitik restriktiver. Denn je stärker die Rechte der Bürgerinnen und Bürger ausgeweitet wurden, desto eindeutiger musste definiert werden, wem diese zustanden und wem nicht.

Insgesamt entstand damit ein wachsender Widerspruch zwischen der Einbindung in den Wohlfahrtsstaat und den politischen Ausschlussmechanismen. Dieser Widerspruch veränderte die politische Landschaft grundlegend. Je stärker die Migration wuchs, desto deutlicher wandten sich grosse Teile der Arbeiterschaft von den Linksparteien, die den Ausbau des Sozialstaates erkämpft hatten, ab und den Rechtspopulisten, die gegen die Migration kämpfen, zu. Die Arbeiter waren

20 Hammar, Thomas: Democracy and the Nation State: Aliens, Denizens and Citizens in a World of International Migration, Aldershot: Avebury1990; Bauböck, Rainer: »Gleichheit, Vielfalt und Zusammenhalt«, in: Volf, Patrick/ders.: Wege zur Integration, Klagenfurt/Celovec: Drava 2001, S. 11-41, hier S. 26.

21 Wimmer, Andreas: »Binnenintegration und Aussenabschliessung. Zur Beziehung zwischen Wohlfahrtsstaat und Migrationssteuerung in der Schweiz des 20. Jahrhunderts«, in: Bommes, Michael/Halfmann, Jost: Migration in nationalen Wohlfahrtsstaaten, Theoretische und vergleichende Untersuchungen. Osnabrück: Rasch 1998, S. 199-221. 
lange Zeit selber Ausgeschlossene gewesen und hatten sich ihre politische und soziale Integration hart erkämpft. Immer mehr von ihnen wie auch viele Angehörige des diffusen „Mittelstandes“ sind heute der Meinung, die Migrierenden würden von den sozialen Institutionen profitieren, ohne zu ihnen beigetragen zu haben, während sie selber Gefahr laufen würden, wegen der wachsenden Konkurrenz ihre Arbeitsplätze wie auch ihre sozialen Rechte zu verlieren. ${ }^{22}$

Eine Liberalisierung des Migrationsregimes ist historisch gesehen daher eher mit einer Schwächung sozialstaatlicher Integration verbunden. In den letzten Jahren konnte man beobachten, wie dieser für Nationalstaaten postulierte Effekt auch bei der EU spielte, allerdings in der umgekehrten Richtung. Der Ausbau der ökonomischen, politischen und sozialen Vernetzung zwischen den EU-Mitgliedern fördert (wie vor einem Jahrhundert bei der Entstehung der Nationalstaaten) interne Mobilität unter dem Grundsatz der Personenfreizügigkeit, zugleich aber auch die Abschottung nach aussen. Die EU versucht immer intensiver, ihre Aussengrenzen abzusichern, um die innere Mobilität zu schützen.

Viele europäische Gesellschaften haben im Übergang zur nationalstaatlichen und industriellen Moderne die bürgerlichen und politischen Rechte von einer lokalen bzw. regionalen Ebene auf eine nationale übertragen. Sie müssen sich nun wohl Gedanken machen, wie Partizipation in einer zumehend mobilen und interdependenten Gesellschaft aussehen könnte. Genügt in einer Welt, in der immer mehr Menschen in zwei oder mehr Staaten Teile ihres Lebens verbringen, der Nationalstaat als Aktionsradius der Demokratie? Das bisherige Versprechen dieses Nationalstaates ist die exklusive Deckungsgleichheit von Gesellschaft, Politik und Territorium, also von sozialem, politischem und geografischem Raum. Nun haben wir es spätestens seit dem Ende des Zweiten Weltkriegs bei einer zunehmenden Anzahl grenzüberschreitender Prozesse mit einer Emanzipation des sozialen vom geografischen Raum zu tun, so dass beide immer weniger übereinstimmen. Weit auseinander liegende geografischen Räume werden durch Technik und Kommunikation miteinander verbunden. Gleichzeitig werden die sozialen Räume komplexer: Einerseits werden sie immer heterogener, verschachtelter und durchmischter, andererseits weiten sie sich auch aus, bilden geografisch nicht mehr verbundene Einheiten, die weit entfernt lebende Communities durch moderne Technik einen gemeinsamen sozialen Raum ohne geografische Grundlage

22 Häusermann, Silja: »Der Preis des Erfolgs. Die Linke verliert mehr und mehr Wähler an rechtsnationale Parteien. Wie lässt sich dieser Trend umkehren?«, in: NZZ, 15.2.2017; Banting, Keith/Kymlicka, Will (eds.): Multiculturalism and the Welfare State. Recognition and Redistribution in Contemporary Democracies, Oxford: OUP 2009. 
herstellen lassen. ${ }^{23}$ Wenn soziale Gruppen aber losgelöst vom geografischen Raum existieren können, ist räumliche Nähe keine notwendige Bedingung für gemeinsame politische Rechte mehr. Der Nationalstaat verliert als Referenzrahmen für wirtschaftliche, soziale und politische Prozesse an Bedeutung. Wie aber könnten bürgerschaftliche Modelle jenseits eines ausschliesslich flächenräumlich gedachten Staates funktionieren?

Bevor man sich dieser Frage zuwendet, muss geklärt werden, wie Staatszugehörigkeit eigentlich definiert wird. Ihr zentrales Element ist das Bürgerrecht. In Ländern wie der Schweiz, aber auch in vielen anderen Staaten ist ein immer höherer Anteil der Bevölkerung eingewandert. Gegenwärtig liegt in der Schweiz der Ausländeranteil bei einem Viertel der Bevölkerung. Mehr als ein Drittel hat einen so genannten Migrationshintergrund, d. h. mindestens ein Elternteil ist eingewandert. Mehr als 40 Prozent der Ehschliessungen sind binational. ${ }^{24}$ Das Bild der Nation als eine homogene Gruppe von Menschen, die sich durch eine gemeinsame Geschichte verbunden ist und die auf der Basis dieser gemeinsamen Herkunft Bürgerrechte besitzt, ist daher immer weniger zutreffend. Das ius sanguinis, wie dieses Abstammungsprinzip zur Begründung staatlicher Zugehörigkeit genannt wird, macht daher immer weniger Sinn als Legitimation der Staatszugehörigkeit, auch wenn es für weite Teile Europas noch immer bestimmend ist. Zwar besteht schon heute die Möglichkeit, eingebürgert zu werden, ohne diese Abstammung vorweisen zu können, aber dieses Prozedere gilt als Ausnahme, nicht als Prinzip staatlicher Zugehörigkeit.

Allerdings ist die breite Durchmischung der Bevölkerung nicht ganz so neu, wie sie manchmal dargestellt wird. Bereits der sich rasch modernisierende schweizerische Bundesstaat des ausgehenden 19. und frühen 20. Jahrhunderts zum Beispiel sah sich mit einer wachsenden Einwanderung konfrontiert, so dass 191415 Prozent der Bevölkerung Ausländer waren, in Städten wie Lugano 30.8, Basel 37.6 und Genf 40.4 Prozent. Dass dieser Anteil nicht noch grösser war, dafür sorgte die damalige Politik der raschen Einbürgerung. Bis nach dem Ersten Weltkrieg betrug die Wartefrist für den entsprechenden Antrag gerade mal zwei Jahre. Vor allem liberale Kreise störten sich daran, dass ein immer grösserer Teil der Bevölkerung von der politischen Partizipation ausgeschlossen war. Manche verlangten sogar eine Zwangseinbürgerung der zweiten Generation auf der Basis des

23 Pries, Ludger: »The Approach of Transnational Social Spaces: Responding to New Configurations of the Social and the Spatial«, in: Ders. (ed.): New Transnational Social Spaces. International Migration and Transnational Companies, London: Routledge 2001, pp. 3-33.

24 http://www.binational.ch/de/?Vorbemerkungen Allgemeines (16.3.2019). 
ius soli, welches den Geburtsort als entscheidend ansieht. Die klassischen Einwanderungsländer wie die USA, Kanada, Australien und Neuseeland bürgerten ohnehin schnell ein, ihr Verständnis von Zugehörigkeit beruhte immer schon auf dem ius soli. Die europäischen Staaten haben diese Möglichkeit aber nie akzeptiert.

Was sich hingegen immer stärker durchsetzt, sind Doppel- oder Mehrfachbürgerschaften. ${ }^{25}$ Lange Zeit war der Widerstand dagegen gross. Vor allem Auswanderungsländer fördern aber die doppelte Staatsbürgerschaft immer stärker, weil sie befürchten, sonst die Verbindung zu ihren ausgewanderten Bürgerinnen und Bürgern zu verlieren. In diversen Ländern sind Auslands-Staatsangehörige mit eigenen Sitzen im Parlament vertreten. Man spricht auch von „extraterritorialer Staatsbürgerschaft".${ }^{26}$ Noch fehlt das Pendant zu dieser Entwicklung, nämlich Sitze für Einwanderinnen und Einwanderer im Parlament der Einwanderungsstaaten.

Wenn Statistiken über die Bevölkerung einzelner Staaten veröffentlicht werden, dann sind diese in der Regel binär aufgeteilt in Inländer und Ausländer. Nicht berücksichtigt werden die doppelten und mehrfachen Staatsbürgerschaften. Bezieht man diese in die Berechnungen ein, entsteht ein neues Bild von Gesellschaft: Nicht das Bild eines Entweder - Oder, Inländer - Ausländer, sondern das Bild eines zunehmenden Sowohl als Auch, sowohl Inländer als auch Ausländer. 2016 besassen 23,9 Prozent aller Schweizerinnen und Schweizer, die über 15 Jahre alt sind, eine mehrfache Staatsbürgerschaft, bei den in der Schweiz lebenden Personen waren es 13 Prozent. Von den Schweizern im Ausland können sogar drei Viertel auf mehrere Pässe zurückgreifen. Durch die hohe Anzahl binationaler Ehen wächst die Zahl der Mehrfachbürgerinnen und -bürger qua Geburt sehr schnell.

25 Blatter, Joachim/Sochin D'Elia, Martina/Buess, Michael: Bürgerschaft und Demokratie in Zeiten transnationaler Migration: Hintergründe, Chancen und Risiken der Doppelbürgerschaft. Studie im Auftrag der EKM, Bern: BBL 2018; vgl. auch Faist, Thomas/Kivisto, Peter (eds.): Dual Citizenship in a Globalizing World, Basingstoke: Palgrave Macmillan 2007.

26 Bauböck, Rainer: »The Rights and Duties of External Citizenship«, in: Citizenship Studies 13/5 (2009), pp. 475-499; Collyer, Michael: »A Geography of Extra-territorial Citizenship: Explanations of external Voting«, in: Migration Studies 2/1 (2014), pp. $55-72$. 


\section{AKTIONSRADIEN DER DEMOKRATIE}

Die heutige Verteilung der Rechte schliesst einen wesentlichen Teil der Bewohnerinnen und Bewohner eines Staates von einer politischen Beteiligung aus. Wie hoch darf deren Anteil sein, ohne dass die Demokratie ihre Legitimation verliert? Demokratietheoretisch lässt sich kaum begründen, dass wesentlichen Anteilen der Bevölkerung entsprechende Rechte vorbehalten werden. Demokratie basiert auf einer universalistischen Logik der Zugehörigkeit, nationalstaatliche Zugehörigkeit hingegen auf einer exkludierenden. Hier liegt ein wesentlicher Widerspruch nationalstaatlicher Demokratie, der sich mit zunehmender Mobilität stärker akzentuiert. $^{27}$

Kann im Extremfall eine Minderheit Demokratie praktizieren und die Mehrheit ausschliessen? Die Möglichkeit der schnelleren demokratischen Beteiligung unabhängig von der Staatsbürgerschaft auf zunächst lokaler und regionaler Stufe könnte hier einen ersten Schritt darstellen. In vielen Staaten findet deshalb eine intensive Debatte über neue Formen der Bürgerschaft und der Partizipation statt. In der französischen Sprache gibt es einen Ausdruck für diese grundlegende Form von Partizipation: citoyenneté. Als citoyen, citoyenne gelten nicht einfach Personen, die zufällig Bürgerin oder Bürger eines Landes sind, sondern Personen, die sich kümmern, die mit ihrer Haltung und ihrem Engagement diese Gesellschaft erst ausmachen, die sich verantwortlich fühlen für ihr Umfeld und ihre Umwelt. ${ }^{28}$

In einer historischen Betrachtung stellen wir fest, dass tatsächlich immer weitere Gruppen in den politischen Entscheidungsprozess einbezogen worden sind. Zudem ist auch eine räumliche Expansion politischer Rechte zu erkennen. Lange Zeit waren es die Gemeinden und Städte, die Rechte vergaben, sei das in der griechischen Polis, sei das in den Städten und Gemeinden des Mittelalters und der Frühen Neuzeit. Diese lokalen Rechte spielen in der Entwicklung hin zur Moderne eine zentrale Rolle. Mit dem Nationalstaat entstand dann die Gleichsetzung von staatlicher Zugehörigkeit in Form der Staatsbürgerschaft und der Ausübung der demokratischen Rechte. Anders als dieses heute dominierende Verständnis von

27 Perchinig, Bernhard: »Migration, Integration und Staatsbürgerschaft - was taugen die Begriffe noch?«, in: Langenthaler, Herbert (Hg.): Integration in Österreich. Sozialwissenschaftliche Befunde, Innsbruck: Studienverlag 2010, S. 13-33; Bauböck, Rainer (ed.): Migration and Citizenship. Legal Status, Rights and Political Participation, Amsterdam: AUP 2006; Isin, Engin F./Saward, Michael (eds.): Enacting European Citizenship, Cambridge: CUP 2013.

28 Vgl. auch Serrut, Louis-Albert: De la citoyenneté. Histoire et émergence d'un concept en mutation, Paris: Editions du Cygne 2016. 
Bürgerschaft als spezifische juristische Rechte und Pflichten umfassende $\mathrm{Zu}$ schreibung an ein einzelnes Subjekt versteht ein Teil der neueren Citizenship-Forschung Bürgerschaft in Anlehnung an citoyenneté-Konzepte zunehmend als soziale Praxis, durch welche Individuen und Kollektive ihre Rechte behaupten, erkämpfen oder verlieren können: Praxen, die Engin F. Isin einerseits als (bewusste) „acts of citizenship“ und andererseits als (unreflektierte) habituelle Praktiken sieht. ${ }^{29}$ Die gesellschaftliche Reproduktion des bürgerschaftlichen Status wird in den alltäglichen sozialen Praktiken verortet und kann als doing citizenship verstanden werden. Bürgerschaft geht hier also von Partizipation und Engagement, aber auch von der Vielfalt der beteiligten Gruppen aus. Grade für vielfältige Gesellschaften bleibt aber die zentrale Frage, ob es ihnen gelingt, eine ,inclusive political culture" herzustellen. ${ }^{30}$ Lange wurde auf der staatlichen Ebene die Einheit und Gleichheit der Menschen hervorgehoben, in den Städten hingegen liegt die Betonung auf Diversität und Differenz. ${ }^{31}$ Städte sind seit jeher Orte der Vielfalt, sie organisieren ,the being together of strangers“" ${ }^{\text {32 }}$, sind aber gleichzeitig auch immer Orte, an denen ein intensiver Kohäsionsprozess dafür sorgt, dass sie als Strukturen funktionieren. Bürgerschaft wird in diesen Ansätzen also nicht ausschließlich über die Rechte definiert, die der Staat garantiert, sondern über die Handlungen, Verhaltensweisen und Praktiken der Menschen, nicht als statisches, sondern als dynamisches Konzept verstanden, nicht als etwas, das wir qua Geburt haben, sondern als etwas, das wir erarbeiten. Allerdings stellt sich sogleich die Frage, wer das Recht hat darüber zu entscheiden, wer an einem gewissen Ort leben, wer sich in bestimmten Städten und Staaten aufhalten darf. So sehr Urban Citizenship-Theorien davon ausgehen, dass alle, die in einer bestimmten Stadt sind, ungeachtet ihrer rechtlichen Stellung Möglichkeiten der Partizipation haben sollten, so sehr halten die Staaten daran fest, Einreisen kontrollieren und sanktionieren zu können. Selbst an Orten grosser Offenheit, was neue Partizipationsformen betrifft, ist keine Entwicklung hin zur prinzipiellen Aufgabe der Idee der Staatsbürgerschaft, die nur unter gewissen Bedingungen erworben werden kann, zu verzeichnen. Dass an manchen Orten zwischen der urbanen und nationalen Ebene noch weitere Ebenen liegen (Kantone, Distrikte, Bundesländer), macht die

29 Isin, Engin F./Nielsen, Greg M. (eds.): Acts of Citizenship, London: Zed 2008; Smith, Michael P./McQuarrie, Michael (eds.): Remaking Urban Citizenship: Organizations, Institutions, and the Right to the City, New Brunswick: Transaction 2012.

30 Zukin, Sharon: The Cultures of Cities, Malden: Blackwell 1995, p. 44.

31 Sandercock, Leonie: Towards Cosmopolis: Planning for Multicultural Cities, New York: John Wiley 1998, p. 183.

32 Young, Iris M.: Justice and the Politics of Difference, Princeton: PUP 1990, p. 237. 
Auseinandersetzung nicht einfacher. Dennoch ist die Frage der Partizipation innerhalb dieser komplexen Konstellationen weiter zu denken und weiter zu entwickeln.

Weitergedacht werden muss auch, wie staatsbürgerschaftliche Modelle jenseits des flächenräumlichen Staates funktionieren könnten. Die EU hat mir ihrer Bürgerinitiative, die in Wirklichkeit einem Petitionsrecht entspricht, ein solches Instrument etabliert, das aber nicht wirklich als Agens demokratischer Mitbestimmung funktioniert. ${ }^{33}$ Solche Bestrebungen müssen erweitert werden, etwa auf der Ebene grenzüberschreitender Entscheidungsprozesse, da diese häufig Bewohnerinnen und Bewohner einer Region gemeinsam betreffen, selbst wenn sie in verschiedenen Staaten leben. Vorstellbar wäre auch, dass man Angehörigen benachbarter Staaten Sitze in nationalen Parlamenten zugesteht und länderübergreifende regionale Parlamente schafft. Und schliesslich sind auch transstaatliche Konzepte der politischen Partizipation notwendig zur Sicherung demokratischer Strukturen in einer transnationalen Gesellschaft. ${ }^{34}$

Auf allen Ebenen von lokal bis global muss Partizipation neu gedacht, müssen multilevel oder multilayered citizenship regimes entwickelt werden. ${ }^{35}$ Staatliche Zugehörigkeit und die mit ihr verbundenen Rechte werden sich von der Exklusivitätsvorstellung, die ihnen heute noch innewohnt, lösen und sich Richtung multipler Zugehörigkeiten wandeln, auch wenn eine solche Entwicklung aktuell eher utopisch wirkt. Die Demokratien stehen an vielen Orten unter dem Druck illiberaler Forderungen, welche die enge Verflechtung von Demokratie, Rechtsstaatlichkeit und Menschenrechten nicht akzeptieren. Ein Rückzug auf nationalstaatliche Konzepte dürfte keine ausreichende Antwort auf diese Herausforderung bieten. Was also verhandelt werden muss, sind grundlegende Fragen der

33 Hieber, Thomas: Die Europäische Bürgerinitiative nach dem Vertrag von Lissabon Rechtsdogmatische Analyse eines neuen politischen Rechts der Unionsbürger. Diss. St. Gallen 2013.

34 Kivisto, Peter/Faist, Thomas: »The Boundaries of Citizenship: Dual, Nested, and Global«, in: Dasgupta, Samir/Nederveen Pieterse, Jan (eds.): Politics of Globalization. New Delhi: Sage 2009, pp. 356-376; Linklater, Andrew: »Cosmopolitan Citizenship«, in: Isin, Engin F./Turner, Bryan S. (eds.): Handbook of Citizenship Studies. London: Sage 2002, pp. 317-332.

35 Maas, Willem: »Multilevel Citizenship«, in: Shachar, Ayelet et al. (eds.): The Oxford Handbook of Citizenship (OUP, erscheint 2017) pp. 644-668; Yuval-Davis, Nira: »The Multi-layered Citizen«, in: International Feminist Journal of Politics, 1/1 (1999), pp. 119-136. 
gesellschaftlichen Kohäsion und Partizipation im Zeitalter der Globalisierung, so wie sie mit der Bildung der Nationalstaaten im 19. Jh. neu ausgehandelt wurden. Weil aber heute zukunftsweisende Perspektiven weitgehend fehlen (damals öffneten Liberalismus und Sozialismus neue Horizonte), das Partizipations-Narrativ des globalen Zeitalters noch nicht gefunden ist, verharren die westlichen Gesellschaften im Zustand der Abwehr und der Regression und fokussieren primär auf Migrierende als Verkörperung des Wandels, ohne die Fragen der gesamtgesellschaftlichen Transformation wirklich anzupacken. 